\title{
Experiencing Various Color Models on Colored Images
}

\author{
Noor A. Ibraheem \\ Computer Science Department \\ College of Science for Women \\ University of Baghdad \\ Baghdad, Iraq
}

\author{
Mokhtar M. Hasan \\ Computer Science Department \\ College of Science for Women \\ University of Baghdad \\ Baghdad, Iraq
}

\begin{abstract}
Colors are important for human communicating with the daily encountered objects as well as his species, these colors should be represented formally and numerically within a mathematical formula so it can be projected on device/ computer storage and applications, this mathematical representation is known as color model that can hold the color space, by the means of color's primary components (Red, Green, and Blue) the computer can visualizes what the human does in hue and lightness. In this work a review of most popular color models are given (which are RGB, CMY, HSV, and $\mathrm{YCbCr}$ ) with the explanation of the components, color system, and transformation formula for each other, application areas and usages are also included. Comparison between these different color models is performed by applying Signal to noise Ratio (SNR) metric to indicate the best color models. Results analysis shows the RGB has better results according to SNR measure.
\end{abstract}

\section{General Terms}

Color Model, RGB, CMY, HSV, YCbCr, skin color detection, segmentation.

\section{Keywords}

Color Model, RGB, CMY, HSV, YCbCr, skin color detection, segmentation.

\section{INTRODUCTION}

The field of digital image processing has grown in extensive manner in our ear especially with the significant increase in life applications such as weather prediction relying on satellite image, diagnosis of diseases depending on biomedical image, security purposes using object tracking, besides communications, intelligent transportation systems, television etc. [1]. Each field of digital image processing demanded a digital colored images format, so the need to understand the components of colors and how they represent the entire color will easy and simplifies that work [3].

However, the main two steps of digital image processing is to enhance image information according to human image understanding, and to process these information formally in a suitable application area [2].

Color model is a method for specifying colors in a mathematical way; it consists of three dimensional coordinates (components) where each color is constructed by a point, for human vision system, the eye is sensitive to light waves' colors that have low intensity so it can identify colored images. The red, green and blue colors are called the primary colors while cyan, yellow and magenta are known as secondary colors which can be gained by combining two primary colors, for example cyan can be obtained by mixing blue and green [4][5].

\section{COLOR MODEL}

The color model (also called color space) is the formal representation of the set of the three colors that format the vector space. It can be defined as the way to identify color in which human visualize color through its components such as; hue, and brightness [6][7].

There are two types of color models:

1. Image color models: This usually considered images representation and image's information quality such as RGB, CMY, HSI etc.

2. Video color models: This kind of models considered video representation and concentrated on minimizing the band width for video signals transferring such as YIQ ,YUV ,YCbCr etc. [3]

Moreover, there are three basic color models families can be categorized [1]:

1. Colorimetric color models, this metric relies on physical measurements such as the CIE color model that considered as the initial points for other models.

2. Psychophysical color models, this models are the based for human color perception, these models depends on the subjective observation criteria and comparative references such as Munsell color space) or representation of the human perception of color such as hue, saturation and lightness.

3. Physiologically inspired color models, that are mainly relies on the three primaries, Red, Green, and Blue (RGB) color space used in computer hardware.

\subsection{RGB Color Model}

RGB color model is the additive color model that are represented by three primary colors, in which Red, Green and Blue light waves are combined all together to produce a broad color. RGB is device dependent color model and the range of its components is [0-255] for the three basic colors [3].

The RGB color model is an additive color model, that are usually used in various transmission and communication media that transmit light such as computer monitors, phones, and digital media that emit color as RGB light [5]. Figure 1 shows the representation of the additive colors and their combination produces the white color [8] 
Table 1: Color models with the effective parameter and their advantages and disadvantages [6].

\begin{tabular}{|c|c|c|c|c|}
\hline Model & Parameters & $\begin{array}{c}\text { Effective } \\
\text { Parameter }\end{array}$ & Advantages & Disadvantages \\
\hline RGB & $\begin{array}{l}\text { Red, } \\
\text { Green, } \\
\text { Blue. }\end{array}$ & $\mathrm{R}, \mathrm{G}, \mathrm{B}$ & $\begin{array}{l}\text { (1) No transformations required to display } \\
\text { information on the screen, for this reason } \\
\text { it considered as the base color space for } \\
\text { various applications. } \\
\text { (2) Used in video display because of } \\
\text { additive property. } \\
\text { (3) Considered as computationally } \\
\text { practical system. }\end{array}$ & $\begin{array}{l}\text { (1) Non useful for objects specification } \\
\text { and recognition of colors. } \\
\text { (2) Difficult to determine specific color } \\
\text { in RGB model. } \\
\text { (3) RGB reflects the use of CRTs, since } \\
\text { it is hardware oriented system. }\end{array}$ \\
\hline $\mathrm{CMY}(\mathrm{K})$ & $\begin{array}{l}\text { Cyan, } \\
\text { Magenta, } \\
\text { yellow, and } \\
\text { Black }\end{array}$ & - & $\begin{array}{l}\text { (1) Commonly used for production printer } \\
\text { color. }\end{array}$ & $\begin{array}{l}\text { (1) Since it is a subtractive model, the } \\
\text { components are pigments or inks not } \\
\text { colors. }\end{array}$ \\
\hline HSV & $\begin{array}{l}\text { Hue, } \\
\text { Saturation, } \\
\text { Value }\end{array}$ & V & $\begin{array}{l}\text { (1) HSV colors defined easily by human } \\
\text { perception not like RGB or CMYK. }\end{array}$ & $\begin{array}{l}\text { (1) Undefined achromatic hue points are } \\
\text { sensitive to value deviations of RGB and } \\
\text { instability of hue, because of the angular } \\
\text { nature of the feature. }\end{array}$ \\
\hline $\mathrm{YCbCr}$ & $\begin{array}{l}\mathrm{Y} \\
\text { Luminance, } \\
\text { (Cb and } \\
\mathrm{Cr} \text { ) are } \\
\text { chrominanc } \\
\text { e } \\
\text { blue } \\
\text { difference, } \\
\text { and red } \\
\text { difference }\end{array}$ & $\mathrm{L}$ & $\begin{array}{l}\text { (1) Perfect in image compression. } \\
\text { (2) Used in saving images as a file format } \\
\text { for image. } \\
\text { (3) Y luminance can be use separately for } \\
\text { storage in high resolution and the } \\
\text { chromaticity components treated separately } \\
\text { to improve the performance. }\end{array}$ & $\begin{array}{l}\text { (1)The color range is restricted in the } \\
\text { color TV images because of the } \\
\text { information compression required for the } \\
\text { displayed image. } \\
\text { (2) The displayed color depends on the } \\
\text { primaries RGB that displayed the signal. }\end{array}$ \\
\hline
\end{tabular}

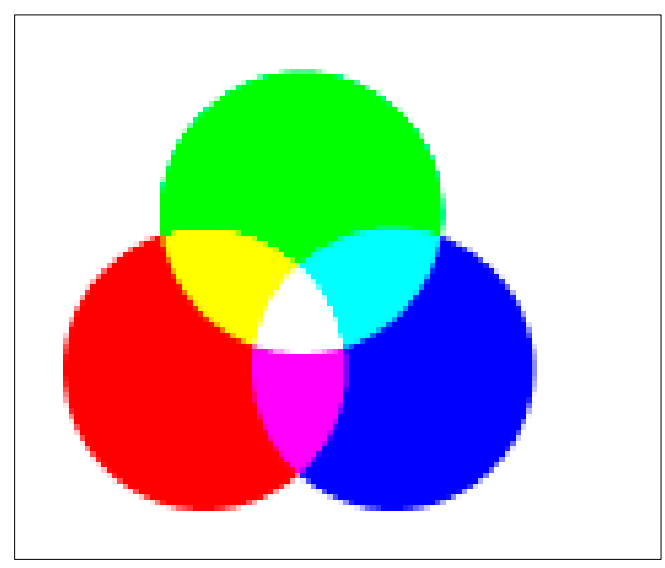

Fig 1. RGB color model

\subsection{CMY Color Model}

CMY color model is the subtractive colors and it is device dependent color model, these colors are Cyan, Magenta and Yellow [3]. These colors considered as the subtractive colors where their combination produces the black color, the range of values for each color is in the range [0-255]. The conversion between CMY and RGB color models is represented by subtracting each color in RGB with its corresponding color in CMY color model; reference [6] shows the conversion formula between these models, Figure 2 explains this color model.

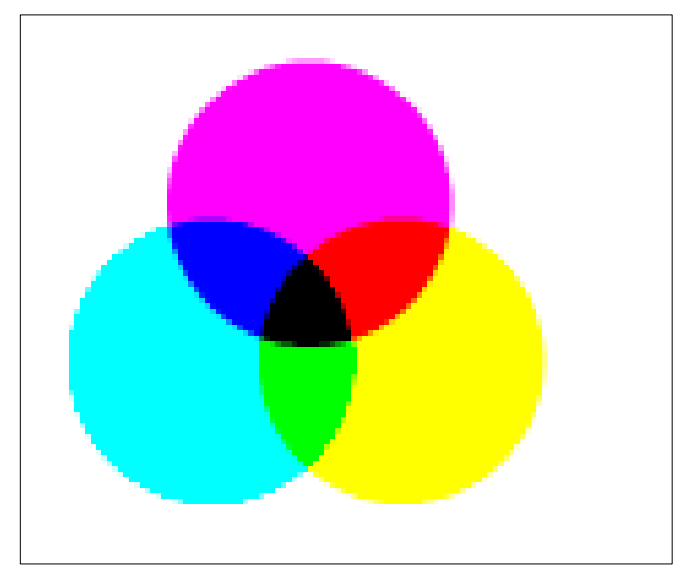

Fig 2. CMY color model.

\subsection{The YIQ Color Space}

YIQ color model adopted in 1950 by the National Television Standard committee (NTSC) where the RGB inputs from the camera are converted to a luminance (Y) and two chrominance information I and Q. YCbCr color model used for digital video, also extensively used in European TV signal [6]. YCbCr color model components are Y for luminance and Chroma components where $\mathrm{Cb}$ and $\mathrm{Cr}$ components, transformation from RGB to $\mathrm{YCbCr}$ color model is explained in [6], Figure 3 shows this color model [9]. 


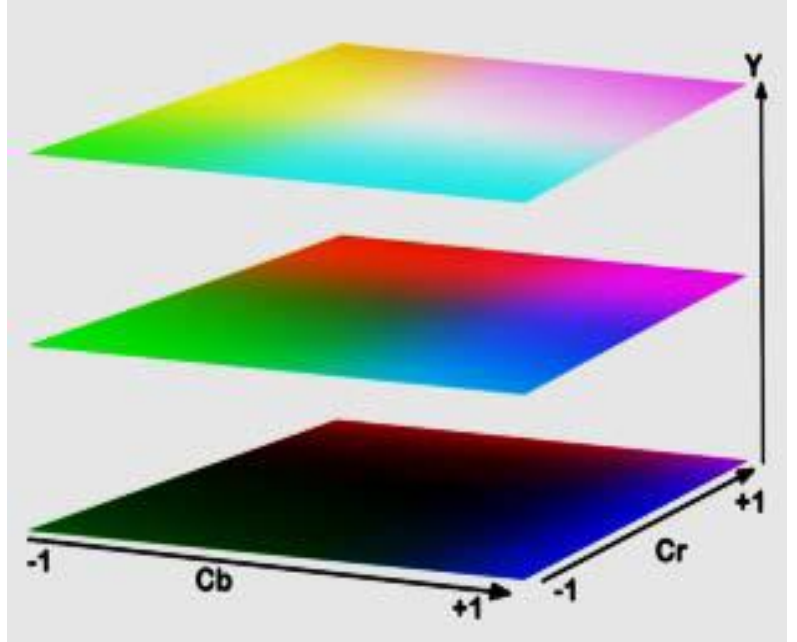

Fig 3. YCbCr color space.

\subsection{The HSV Color Model}

HSV and HSL color models are the two common cylindricalcoordinate representations, developed in the 1970s for computer graphics applications, mainly used in image software, and less used in computer vision [1]. HSV color model stands for hue, saturation, and value, and sometimes known as HSB (B for brightness), or HSI (I for intensity). The conversion between each of RGB and HSV can be found and applied in references [6-11]. Figure 4 demonstrates HSV color model.

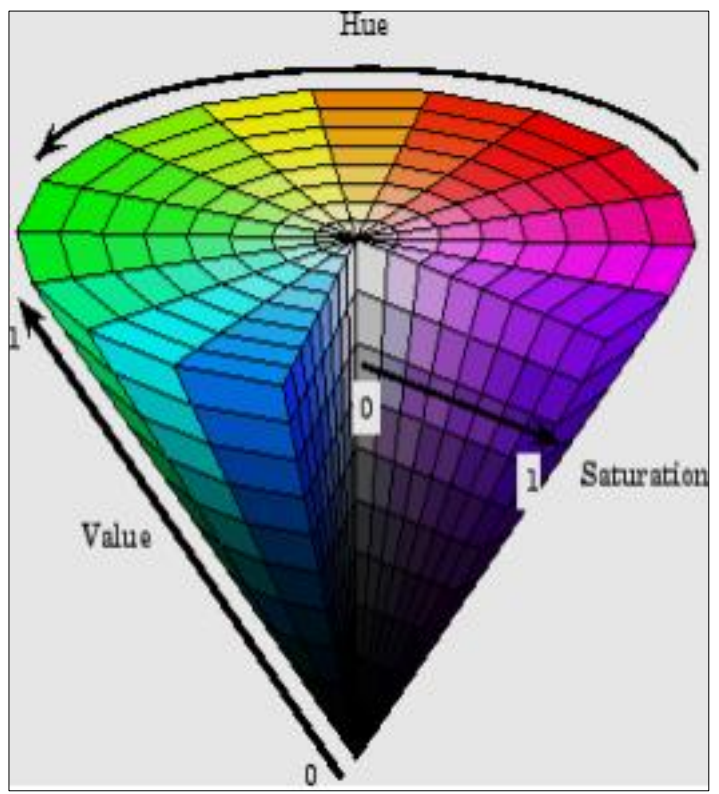

Fig 4. HSV color model [6].

\section{SIGNAL TO NOISE RATIO (SNR)}

The Signal to Noise Ratio (SNR) metric used to predict the performances of low light conditions [10][11], for the low light, image quality is mainly rely on the light level. The SNR is a function of the light level, for images quality measure, a comparison between the pixels of the compared images is calculated. The formula of SNR is computed by calculating the means and standard deviations for each color components of the color model.

$$
\mathrm{SNR}=20 \log _{10}\left(\frac{\mu_{\mathrm{R}}+\mu_{\mathrm{G}}+\mu_{\mathrm{B}}}{\sqrt{\sigma_{\mathrm{R}}^{2}+\sigma_{\mathrm{G}}^{2}+\sigma_{\mathrm{B}}^{2}}}\right)
$$

Where $\mu_{\mathrm{R} ; \mathrm{G} ; \mathrm{B}}$ and $\sigma_{\mathrm{R} ; \mathrm{G} ; \mathrm{B}}$ are the means and standard derivations of each color model.

In this metric, the high value of SNR, the more accepted and correct result.

\section{RESULTS}

As demonstrated by the following Table 2 which illustrated the application of RGB, HSV, YCbCr and CMY color models, the first column is the original mage which is in RGB color model, the second column represents the conversion to HSV color model, the third column represents the conversion to $\mathrm{YCbCr}$ color model, and the last column represents the conversion to CMY color model.

The comparison between various color model types is done by applying (SNR) metric. The comparison has been applied for each color model image separately.

Table 3 shows the implementation of SNR metric for different 4 images used in applying the four RGB, HSV, YCbCr, and CMY color models. The analysis of the results shows that the RGB color model is outperformance on other color models.

\section{CONCLUSIONS}

It has been concluded that each color model is used in different applications depending upon its components and its purpose is to facilitate the specification of colors in some standard generally accepted way. These models used various components of an image to display on specific hardware platform. Researcher can simplify his work by choosing appropriate color model which is the basic for color image representation. Some of the color model characteristics are utilized in different fields of application in our life, such as RGB, YIQ,YCbCr, HSI, HSV, HSL, CIE Lu*v*, and CIE La*b*. Each color model has its own representation space and components, with the ability of transforming from one color pace to another through standard formula. The selection of proper color model for a specific application depends on the properties of the model and the application natures. These color modes are classified mainly into two kinds: device independent and device dependent, further classification is analog use or digital use as well, the application of the color model likewise useful can be found in gesture recognition [12-21] which adopted mainly to extract the background especially cluttered and non-uniform background in which the human face or hand can be easily spotted despite the changing in color pigment of human skin which can be managed by eliminating the chrominance of the modeled color and the luminance will be the judge. 
Table 2: the implementation of the selected color models.

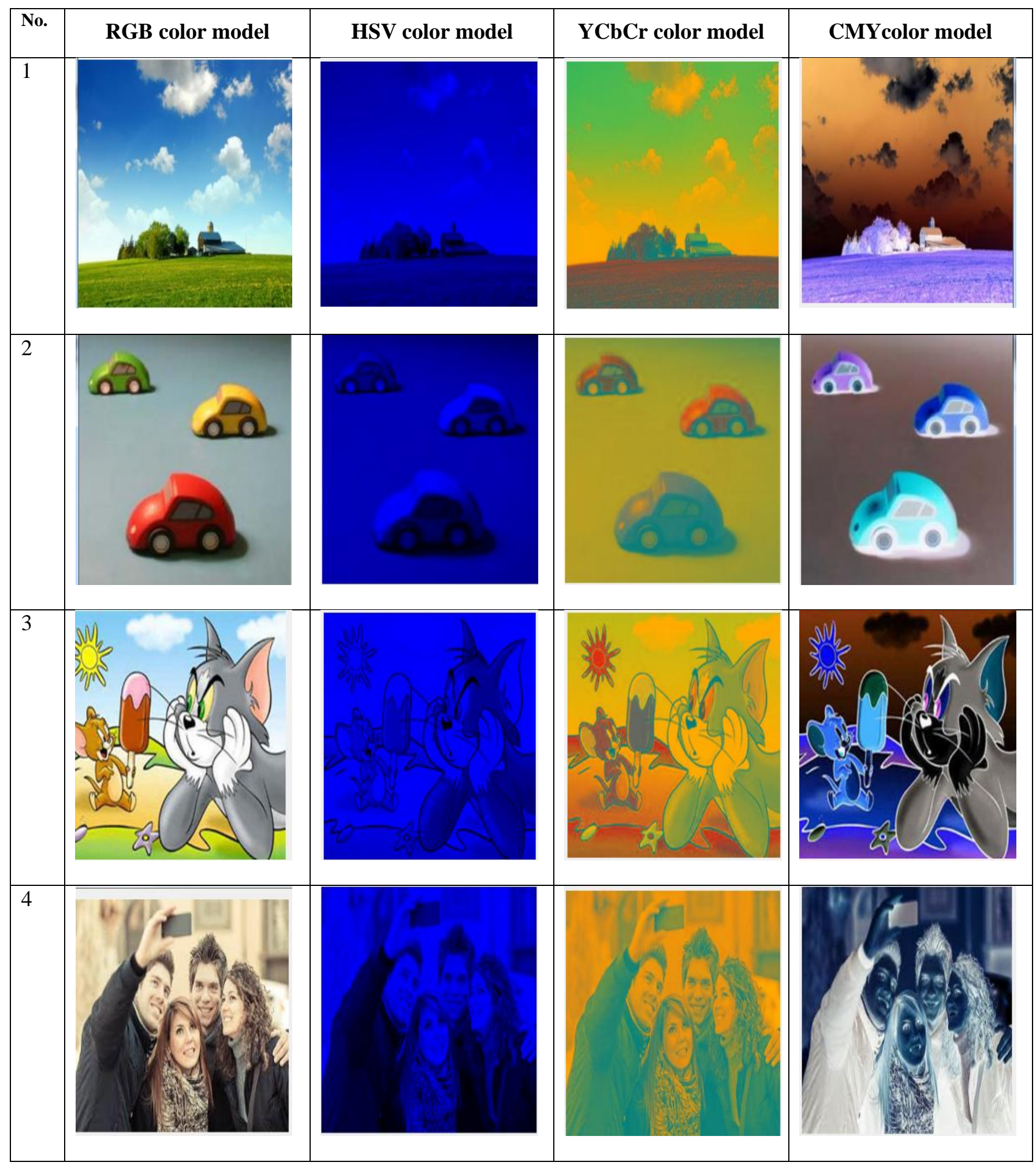

Table 3. SNR metric for RGB, HSV, YCbCr, and CMY color models.

\begin{tabular}{|c|c|c|c|c|}
\hline Picture no. & RGB & HSV & YCbCr & CMY \\
\hline Picture 1 & 43.394 & 34.614 & 36.977 & 43.306 \\
\hline Picture 2 & 41.475 & 33.934 & 33.938 & 41.364 \\
\hline Picture 3 & 43.448 & 35.773 & 36,733 & 43.152 \\
\hline Picture 4 & 42.954 & 38.377 & 33.685 & 42.950 \\
\hline
\end{tabular}




\section{REFERENCES}

[1] K.N. Plataniotis, and A.N. Venetsanopoulos, "Color Image Processing and Applications", book, SpringerVerlag New York, Inc. New York, NY, USA, ISBN:3540-66953-1, 2000.

[2] Gonzalez and Wood Addison, "Digital image processing", Wesley, 1st edition, 1992.

[3] Haneet Kour,"Analysis on Image Color Model", International Journal of Advanced Research in computer and Communication Engineering, Vol.4, issue 12, 2015

[4] Mokhtar M. Hasan, Noor Adnan Ibraheem, "Mixture of GMMs and Mixture of Multiple Histograms for Image Segmentation: A Review", International Journal of Computer Science, vol. 4 , issue 2, number 2, pp.739743, July 2016

[5] Rafiqul Z. Khan,Noor A. Ibraheem, "Novel Segmentation Algorithm based on Mixture of Multiple Histograms", International Journal of Scientific \& Engineering Research (IJSER), (ISSN 2229-5518), Volume 4 (8), pp. 1074-1087, France, August2013.

[6] Noor A. Ibraheem, Mokhtar M. Hasan, Rafiqul Z. Khan, Pramod K. Mishra, "Understanding Color Models: A Review", ARPN Journal of Science and Technology (ISSN: 2225-7217) - (An International Journal), Volume 2 (3), April 2012.

[7] RafiqulZaman Khan, Noor Adnan Ibraheem, "Segmentation Algorithms for Vision Based HCI", Proceedings of the Third Kuwait Conference on eServices and e-Systems (KCESS-2012), ACM Digital Library, New York, , held at Kuwait University, December 18-20, 2012.

[8] Noor A. Ibraheem, Rafiqul Z. Khan, "Multiple Histogram Technique for Robust Skin Color Based Segmentation", American Journal of Engineering Research (AJER), Volume 2(5), (e-ISSN 2229-5518), (pISSN 2320-0936), pp. 50-54, USA, May 2013.

[9] Mokhtar M. Hasan, Noor Adnan Ibraheem, "Melting of Multiple GMM and Multiple Histogram in Segmentation, Gesture Recognition", International Journal of Computer Systems, vol. 3 (7):517-520, India, July, 2016.

[10] Webpage: https://en.wikipedia.org/wiki/Peak_signal-tonoise_ratio.

[11] Fatin E. M. Al-Obaidi, "Image Quality Assessment for Defocused Blur Images", American Journal of Signal Processing, Vol. 5(3), pp. 51-55, 2015. Doi: 10.5923/j.ajsp.20150503.01
[12] Mokhtar M. Hasan, Pramod K. Mishra, "Superior Skin Color Model using Multiple of Gaussian Mixture Model", British Journal of Science, vol. 6(1): 1-14, UK, July 2012

[13] Mokhtar M. Hasan, Ahmed A. Abdul Redha, "Applying Quran Security and Hamming Codes for Preventing of Text Modification", Baghdad Science Journal, University of Baghdad, Iraq, vol.8 (2): 408-418, June 2011.

[14] Mokhtar M Hasan, "New Rotation Invariance Features Based on Circle Partitioning", Journal of Computer Engineering \& Information Technology, vol. 2 (2), USA, July 2013, doi: 10.4172/2324-9307.1000108.

[15] Hasan, M.M., Mishra, P.K., "Direction Analysis Algorithm using Statistical Approaches", SPIE 4th International Conference on Digital Image Processing, 8334-28, 83340L (2012), Malaysia, April 2012, doi: $10.1117 / 12.946046$.

[16] Mokhtar M. Hasan, Pramod K. Mishra, "Novel Algorithm for Skin Color Based Segmentation using Mixture of GMMs", Signal \& Image Processing : An International Journal (SIPIJ), vol. 4 (4): 139-148, India, August 2013, doi: 10.5121/sipij.2013.4412

[17] Mokhtar M. Hasan, Pramod K. Mishra, "Comparative Study for Construction of Gesture Recognition System", International Journal of Computer Science and Software Technology, vol. 4(1): 15-21, January-June 2011.

[18] Mokhtar M. Hasan and Pramod K. Mishra, "Robust Gesture Recognition Using Gaussian Distribution for Features Fitting", International Journal of Machine Learning and Computing, IACSIT Organization, vol. 2(3):266-273, Singapore, June 2012.

[19] Mokhtar M. Hasan, "Object Filling Using Table Based Boundary Tracking”, Journal of College of Education for Women, vol. 28(1): 323-331, March 2017.

[20] RafiqulZaman Khan, Noor Adnan Ibraheem ,"Genetic Shape Fitting for Hand Gesture Modeling and Feature Extraction using Variable Length Chromosome", British Journal of Science, (ISSN 2047-3745), Volume 10 (1), UK, December 2013

[21] Rafiqul Zaman Khan, Noor Adnan Ibraheem "Comparative Study and Analysis for Gesture Recognition Methodologies", International Journal of Advance Research in Science and Engineering, (e-ISSN 2319-8354), (p-ISSN 2319-8346), Volume 2(11), pp.6469, India, November 2013. 